\title{
Axillary hyperhidrosis - topical treatment with aluminium chloride hexahydrate
}

\author{
HAROLD ELLIS \\ F.R.C.S.
}

\author{
JOHN H. SCURR \\ F.R.C.S.
}

Surgical Unit, Westminster Hospital, London SWIP 2AP

\begin{abstract}
Summary
Forty-two patients with axillary hyperhidrosis on the waiting list for surgery were treated with topical saturated solution of aluminium chloride hexahydrate in absolute alcohol. There have been 7 failures. Three patients were unable to cope with the treatment and 4 more experienced severe local irritation or soreness; these 4 were submitted to local surgery. This is a simple and effective treatment for the majority of cases of severe axillary sweating.
\end{abstract}

\section{Introduction}

Axillary hyperhidrosis (excessive sweating) is a relatively common complaint. Patients are socially embarassed by their soaked-through clothes and find that replacement of stained and rotted shirts, blouses and even jackets is a costly exercise (Ellis, 1976). Systemic treatment with anticholinergic drugs is unsatisfactory because of unpleasant side effects. Conventional antiperspirants, although valuable in normal subjects, are ineffective in patients with severe hyperhidrosis. Indeed, these only present to the doctor when all the usual proprietary applications have been tried. The extent of the disability is shown by the fact that these sufferers are only too happy to submit themselves to surgical treatment which is designed to remove the eccrine sweat glands in the axillae, corresponding roughly to the hair-bearing axillary skin, by curettage (Jemec and Hansen, 1978), subcutaneous excision (Skoog and Thyresson, 1962) or excision of an ellipse of the affected skin. This last technique has been found to be most effective by the authors (Ellis, 1977) and they have now performed this operation on some 200 cases. The procedure is not to be undertaken lightly; there may be breakdown or infection of the quite extensive axillary wounds and marked scarring is often seen. In spite of this, patient satisfaction is high and a review of the first 50 patients in the series carried out one year or more after surgery showed that 47 were pleased with the results.

Naturally one would like to avoid surgery, if possible, in what is purely a cosmetic disability. There has been much interest, therefore, in the use of aluminium chloride hexahydrate as a topical appli-8 cation in this condition. This was first reported by 3 . Shelley (1974), who used a $20 \%$ solution in absolute of ethyl alcohol applied at night with an occlusive or dressing. The following year Shelley and Hurley $\mathrm{O}^{\circ}$ (1975) reported successful treatment in 5 patients 0 using this technique. Sneddon (1976) and Price (1977) $\infty$ confirmed satisfactory results with this topical solu-응 tion. Scholes and his colleagues (1978) have recently reported an extensive experience of 65 patients with excellent control in 64. Occlusion of the area $\bigodot_{\mathbb{\Phi}}$ was found to be unnecessary. They advised appli- $\frac{1}{3}$ cation of the $20 \%$ solution in absolute alcohol nightly with a small brush applied to the dried axilda which should not be shaved for $24 \mathrm{hr}$ before and after treatment. Nightly application was continuen. $\odot$ for one week, after which the patient applied the solution only when necessary. After the initial period of nightly treatment the interval between applications $\overline{0}$ varied between 2 days and one year but most patients $\approx$ had to apply the solution once every one to 3 weeks $\stackrel{\mathbb{Q}}{\varnothing}$ to maintain control. About $50 \%$ of the patients $\overrightarrow{\vec{P}}$ experienced some irritation, which was readily 3 relieved by applying a $1 \%$ hydrocortisone cream on the morning after treatment.

Because of these encouraging results, the authors decided to commence a prospective trial of this 3 . technique using patients already on their surgical 0 waiting list with axillary sweating severe enough to 3 warrant axillary skin excision.

\section{Patients and method}

Forty-two patients with a means age of 28 years (range 15-42) were treated. There were 34 females and 8 males in the series, with a mean duration of symptoms of 9 years (range 1-20 years). The solution 5 was prepared by the method described by Scholes et $N$ al. (1978) as an almost saturated solution of alu- N minium chloride hexahydrate in absolute alcohol. O This takes about 3 weeks to dissolve at room temperature. The caustic nature of the solution was well $\frac{\Phi}{\Phi}$ demonstrated in the authors' own study when in the $\stackrel{?}{?}$ initial preparation a stainless steel bucket dissolved! $\square$ In an attempt to reduce the area of application to a 
minimum, the zone of hyperhidrosis was first mapped out using starch iodine powder. Patients were instructed to apply the solution to this demarcated zone nightly for one week, then on alternate nights for a further week and then twice weekly for the remainder of the first month. One per cent. hydrocortisone cream was also provided and patients were told to apply this as frequently as necessary if they experienced any irritation or soreness. The patients were seen after the first week of treatment, at the end of one month and at 6 months.

\section{Results}

Thirty-four patients have been followed-up for 6 months and to date there have been 7 failures. Three patients stopped using the solution, claiming that they were not able to manage the treatment. Four further patients have now been submitted to axillary skin excision, all of whom used the treatment conscientiously and all of whom experienced severe irritation or soreness. The remaining 27 patients described the treatment as highly successful. Twenty patients were able to use the treatment once a week or less, while the remainder used the solution twice or more each week in the same way as they would apply an antiperspirant. Eight patients have been treated for less than 6 months and so far the results seem satisfactory.

The 2 principal side effects are irritation and soreness; indeed, nearly all the patients experienced irritation in the beginning of treatment but this improved over the first few weeks. Fourteen patients experienced continued irritation but only 3 of these considered the irritation to be severe. Six patients complained of soreness and in 3 this was severe. As already stated, 4 patients found the treatment so unpleasant that they opted for surgical cure.

Three early failures were found to be caused by the patients applying the solution in the morning instead of at night but when encouraged to use the treatment at night, all 3 have since been satisfied with the results. Of the 4 patients undergoing surgical treatment, no histological changes in the excised skin or in the axillary glands have been seen but these 3 patients had, however, failed to respond to treatment and this may account for the lack of microscopic changes in the eccrine glands.

\section{Discussion}

The exact mode of action of aluminium chloride is not known. Shelley and Hurley (1975) postulate that there is an action between the aluminium chloride and keratin in the sweat ducts, resulting in their functional closure. There may also be a direct action on the excretory epithelium of the eccrine glands. Whatever the mode of action, the authors can confirm the efficiency of this treatment in many of their patients. The initial irritation is common and persists in a milder form in approximately $30 \%$ of the patients. However, the liberal use of $1 \%$ hydrocortisone cream has allowed most of the patients to continue with treatment and to achieve a successful result. It should be noted that all the patients in this series were prepared to accept surgical treatment and so might be considered severe examples of axillary hyperhidrosis. In spite of this only 4 out of 42 patients to date have required surgical excision, in all cases because of persistent irritation and soreness.

The authors propose to continue a long-term follow-up of these patients to assess the late results of a simple and effective topical treatment for axillary hyperhidrosis.

\section{References}

Ellis, H. (1976) The surgical treatment of hyperhidrosis. Practitioner, 217, 416.

Ellis, H. (1977) Hyperhidrosis and its management. Dermatology Digest, 16, 14.

Jemec, B. \& HaNSEN, B.H. (1975) Follow-up of patients operated on for axillary hyperhidrosis by subcutaneous curettage Scandinavian Journal of Plastic and Reconstructive Surgery, 12, 65,

Price, G.A.R. (1977) Treatment of axillary hyperhidrosis British Medical Journal, 1, 170.

Scholes, K.T., Crow, K.D., Ellis, J.P., Harman, R.R. \& SaIHAN, E.M. (1978) Axillary hyperhidrosis treated with alcoholic solution of aluminium chloride hexahydrate. British Medical Journal, 2, 84.

ShelleY, W.B. (1974) Consultations in Dermatology Vol. 2, p. 259. Saunders, Philadelphia.

Shelley, W.B. \& HuRley, H.J. (1975) Studies on topical antiperspirant control of axillary hyperhidrosis. Acta dermato-venereologica, 55, 241.

Skoog, T. \& Thytresson, N. (1962) Hyperhidrosis of the axilla. A method of surgical treatment. Acta chirurgica scandinavica, 124, 531.

SNedDON, I.B. (1976) Treatment of axillary hyperhidrosis British Medical Journal, 2, 1447. 